\title{
Preference of Food Saltiness and Willingness to Consume Low-sodium Content Food in a Chinese Population
}

$\underline{\text { Pui Hing } \mathrm{CHAU}^{1}}{ }^{*}$, Heidi HY NGAI ${ }^{2}$, Angela YM LEUNG ${ }^{1}$, Siu Fan $\mathrm{LI}^{3}$, Lilian OY YEUNG ${ }^{2}$, Kian Cheng TAN$U^{2}$

1. School of Nursing, The University of Hong Kong, Hong Kong

2. College of Life Sciences and Technology, HKU School of Professional and Continuing Education, Hong Kong

3. The Salvation Army, Hong Kong

* Correspondence to:

$\mathrm{PH}$ Chau

School of Nursing, The University of Hong Kong,

Address: 4/F, William MW Mong Block, LKS Faculty of Medicine, 21 Sassoon Road, Pokfulam, Hong Kong.

Email: phchau@graduate.hku.hk

Telephone: (852) 39176626

Fax: (852) 28726079 


\begin{abstract}
Objective: To compare the preference of food saltiness and the willingness to consume low-sodium food among hypertensive older people, non-hypertensive older people and non-hypertensive young people in a Chinese population.
\end{abstract}

Design: A cross-sectional study based on a quota sample. Three saltiness options (low-sodium, mediumsodium and high-sodium) of soup and bread were offered to each participant who rated the taste of each food on a 5-point Likert scale. Then, the participants rated their willingness to consume the lowsodium content foods on a 5-point Likert scale, given they were informed of the benefit of the lowsodium option. Generalised linear mixed model and multiple linear regression were used to analyse the data.

Setting: Elderly centres and community centres in Hong Kong.

Participants: Sixty hypertensive older people, 49 non-hypertensive older people and 60 nonhypertensive young people were recruited from June to August 2014.

Measurements: The tastiness score and the willingness score were the primary outcome measures. The Chinese Health Literacy Scale for Low Salt Consumption - Hong Kong population (CHLSalt-HK) was also assessed.

Results: The tastiness rating of the high-sodium option of soup was significantly lower than the medium-sodium option $(p<0.001)$, but there was no significant difference between the low-sodium and the medium-sodium options $(p=0.204)$. For bread, tastiness rating of the low-sodium option and the high-sodium option were significantly lower than the medium-sodium option ( $p<0.001$ for both options). The tastiness score of soup did not have significant difference across the groups $(p=0.181)$, but that of bread from the hypertensive older adults $(p=0.012)$ and the non-hypertensive older adults $(p=0.006)$ was significantly higher than the non-hypertensive young adults. Higher willingness rating to consume the low-sodium option was significantly $(p<0.001)$ associated with higher tastiness rating of the lowsodium option of soup and bread, and weakly associated with higher health literacy of low salt intake (soup: $p=0.041$; bread: $p=0.024$ ). Hypertensive older adults tended to be more willing to consume the low-sodium option than non-hypertensive older adults for soup $(p=0.009)$, there was insignificant difference between non-hypertensive older adults and non-hypertensive young adults $(p=0.156)$. For bread, there was insignificant difference in willingness rating to consume low-sodium option $(p=0.375)$.

Conclusion: Older people are at a higher risk of hypertension, reduction of salt intake is important for them to reduce their risk of cardiovascular diseases. There is room for reducing the sodium content of soup, while the sodium in bread should be reduced progressively. Improving the taste of low-sodium food may help to promote reduction in dietary sodium intake.

Keywords: Salt intake, tastiness, willingness, hypertension, health literacy 


\section{Introduction}

Sodium is an essential nutrition component in daily consumption, and a major source of dietary sodium intake is from salt. Both low and excessive sodium intake were shown to associate with higher mortality.[1] In developed countries, excessive sodium intake was more a problem than low sodium intake.[2] The World Health Organization (WHO) recommends a daily salt intake of less than 5g.[3] High salt intake has been shown to be related to elevated blood pressure.[4] In the US, high blood pressure had costed USD46.4 billion direct and indirect costs to cardiovascular diseases and stroke in 2011.[5] A recent review reported a modest reduction in salt intake for at least four weeks was associated with a reduction in blood pressure.[6] Reduction of salt intake was considered as a cost-effective method in reducing blood pressure and its associated risk of cardiovascular diseases.[7]

Concurrent with the hypertension control initiative, there was a decline in stroke mortality in recent decade in the US.[5] On the contrary, it has been reported that the increasing trend in stroke incidence in Hong Kong was concurrent with the increasing trend in hypertension.[8] Near 94\% of the Hong Kong population are of Chinese ethnicity, with $13 \%$ population aged above 65 years.[9] The average salt intake among the healthy adults in Hong Kong increased from 8.0g per day in 1989-1991 to 9.9g per day in 2000-2002.[10] According to the First Hong Kong Total Diet Study conducted in 2010-2011, more than $60 \%$ of the adult population aged $20-84$ years exceeded the WHO recommendation of dietary sodium intake, and on average 6.6g salt was consumed per day.[11] A recent study reported that the Chinese prehypertension postmenopausal women in Hong Kong had a daily salt consumption of 7.8g.[12] It was noted that the estimation methods and reference populations varied in these studies such that it might not be able to conclude for a trend in salt consumption in Hong Kong. Nevertheless, all these figures were consistently above the WHO recommendation of dietary sodium intake.

Reduction of salt intake as a means to population control of hypertension has been drawing increasing attention in Hong Kong. In the Hong Kong Reference Framework for Hypertension Care for Adults in Primary Care Settings, prevention of excess dietary salt intake is included as one of the interventions for reduction and treatment of hypertension in the population.[13] In 2015, the Government establishes

the Committee on Reduction of Salt and Sugar in Food, with a view to reducing the intake of salt and sugar by the public, and the salt and sugar content in food.

Salt reduction interventions are particularly important for older people who have higher risk of hypertension. Reduction of salt intake through individual and food industry interventions should be promoted for population control of hypertension. For example, at the industry level, non-governmental organisations in New Zealand and Australia had been promoting the voluntary reduction of sodium levels in packaged bread products.[14] At individual level, consumption of sodium reduced bread by individuals showed a moderate reduction in urinary sodium and blood pressure.[15]

However, there are various levels of barriers in promoting reduction of salt intake, one of which is the unawareness of the one's unhealthy behaviour. People know certain behaviour is unhealthy, but they often do not aware that they are having such unhealthy behaviour. For example, a multi-national study 
showed that people who consumed more than 6 grams of salt per day claimed their salt intake as satisfactory or even too little.[16]

Furthermore, older people are less sensitive in detecting taste, including savoury taste, than young people.[17, 18] Since the sensation of saltiness is related to the food preference, this loss of sensitivity may cause the older people to have higher salt intake.[19] Some proposed interventions, such as encouraging tasting before addition of salt,[16] may not be effective for the older people given they may have incorrect judgment on the saltiness. Since the older people, such as those in Hong Kong, usually cook for themselves and the young-olds even share the major responsibility in cooking for their family members, their correct judgment on the saltiness is of ultimate importance. Moreover, some interventions to reduce salt in food products in the food industry level may also fail. It is because some people may add salt at the table to compensate the reduction in saltiness of food.[20]

Meanwhile, hypertensive people have been shown to be more likely to be less sensitive to saltiness than normotensive people.[21, 22] Complicated by the loss of salt sensitivity with aging, these hypertensive people may have higher risk of excessive salt intake when they age. This may increase their likelihood of developing cardiovascular diseases. For example, those who experienced heart failure have been shown to have higher salt preference than healthy people.[23]

Sometimes, people know certain behaviour is unhealthy, but they do not have the intention to change their behaviour. For example, people tend to consider taste concerns more than health concerns.[24] It has been shown that some older people, men in particular, were reluctant to reduce salt intake despite they were told about the associated health benefits.[25] The participants opted that the use of salt alternatives could not give the taste as good as that from the use of salt. Another study revealed that despite most people considered reducing salt intake was healthy and important, over one-third had no interest to change their salt intake.[16]

Nevertheless, the historical findings did not show consistent significant relationship between the saltiness preference, aging, and hypertension as revealed by the recent findings.[26-29] Therefore, the current study explored how the older Chinese people's saltiness preference was related to the sodium content in the food. The objective was to compare the preference of food saltiness and the willingness to consume food with low-sodium content among hypertensive older people, non-hypertensive older people and non-hypertensive young people in a Chinese population. By comparing hypertensive older people and non-hypertensive older people, we explored the differences in the outcomes in relation to the hypertension status. By comparing the non-hypertensive older people and non-hypertensive young people, we explored the differences in the outcomes in relation to the age effect.

\section{Methods}

\section{Study Populations}

The target population consisted of adults in Hong Kong. The inclusion criteria were Chinese, Hong Kong residents, aged 18 and above, with no communication problems. The exclusion criteria were any renal illnesses, unstable hypertension (i.e. blood pressure not under control), any strict restrictions in salt or 
sugar intake as advised by health professionals, and food allergy. These people were excluded in order to avoid their possible risk of adverse health effect related to the food consumed.

A quota sample consisted of three different groups of participants were recruited: hypertensive older people, non-hypertensive older people, and non-hypertensive young people. Hypertensive older people was defined as those (i) aged 60 and above, and (ii) had been taking antihypertensive medication for at least 6 months, or not taking antihypertensive medication but with systolic blood pressure above $140 \mathrm{mmHg}$ or diastolic blood pressure above $90 \mathrm{mmHg}$ at recruitment. Non-hypertensive older people was defined as those (i) aged 60 and above, (ii) without any doctor diagnosis of hypertension, (iii) not taking antihypertensive medication, and (iv) systolic and diastolic blood pressure below $140 \mathrm{mmHg}$ and $90 \mathrm{mmHg}$ respectively at recruitment. Non-hypertensive young adults shared the same definition as criteria (ii), (iii) and (iv) of the non-hypertensive older adults, but their age was aged 18 to 45 . Two blood pressure measurements were taken to screen for eligible participants.

\section{Foods Preparation}

Three saltiness options (low-sodium, medium-sodium and high-sodium) of two foods were offered to each participant. Following the food label guidelines issued by the Centre for Food Safety of the Government of Hong Kong, low-sodium option was defined as $<120 \mathrm{mg}$ sodium per $100 \mathrm{~g}$ food, mediumsodium option as $120-<600 \mathrm{mg}$ sodium per $100 \mathrm{~g}$ food, and high-sodium option as $\geq 600 \mathrm{mg}$ sodium per $100 \mathrm{~g}$ food. Two different foods (i.e. corn soup and plain bread) were offered to the same participants so as to eliminate the possible food-specific or brand-specific effect. To protect the participants, the salt content of the food samples did not exceed $1,000 \mathrm{mg}$ sodium per $100 \mathrm{~g}$ food. All food samples were prepared in the teaching kitchen of Department of Nutrition \& Food Management of HKUSPACE Po Leung Kuk Stanley Ho Community College on the day of the fieldwork. Twenty-two people (including four research team members) were involved in piloting the taste of the food, such that all the three options were reasonably tasty and consistent salt concentration in the foods.

The first food offered to the participants was pre-packaged low-sodium corn soup purchasable from supermarket. Since the sodium concentration of the original pre-packaged soup was too low, the research team adjusted it by adding carefully calculated amount of sodium chloride $(0.6 \mathrm{~g})$ to $100 \mathrm{mg}$ sodium per $100 \mathrm{~g}$ food. Then, a medium-sodium option and a high-sodium option were created by adding carefully calculated amount of sodium chloride $(5.4 \mathrm{~g}$ and $12.6 \mathrm{~g}$ respectively) to increase the sodium content to about $300 \mathrm{mg}$ sodium per $100 \mathrm{~g}$ food and $600 \mathrm{mg}$ sodium per $100 \mathrm{~g}$ food respectively. Each pack of soup $(946 \mathrm{ml})$ was heated in a pot to about $70^{\circ} \mathrm{C}$, sodium chloride was added with stirring to ensure uniform sodium concentration. Then, each option of soup was stored in a thermal bottle and transported to the study site. The soup was put in a $30 \mathrm{ml}$ disposable cup labelled with codes to the participants. Throughout the tasting session, the soup was able to maintain a temperature between $43^{\circ} \mathrm{C}$ to $50^{\circ} \mathrm{C}$ at serving.

The second food offered to the participants was home-made bread produced by bread-making machine (model Panasonic SD PM-105), with three saltiness options prepared by adding different amount of salt 
in the preparation of the bread. The ingredients other than salt followed the standard recipe of basic bread available in the recipe of the bread-making machine. The standard recipe of making bread was to use $5 \mathrm{~g}$ of salt to produce $450 \mathrm{~g}$ of bread, which was equivalent to the medium-sodium option with $436.8 \mathrm{mg}$ sodium per $100 \mathrm{~g}$ food. For the low-sodium option, the salt used was reduced to $1 \mathrm{~g}$, which was equivalent to $87.4 \mathrm{mg}$ sodium per $100 \mathrm{~g}$ food. For the high-sodium option, the salt used was increased to $8 \mathrm{~g}$, which was equivalent to $699.0 \mathrm{mg}$ sodium per $100 \mathrm{~g}$ food. Each of the three options of bread was cut into a size of $2 \mathrm{~cm} \times 2 \mathrm{~cm} \times 4 \mathrm{~cm}$ and put in food containers labelled with codes to the participants.

\section{Measure of Food Taste and Willingness to Consume Low-sodium Content Foods}

Saltiness preference was reflected by the perceived taste of the tasted food. During the food tasting session, the participants rated the taste of each food on a 5-point Likert scale, with 1 as "very bad", 2 as "bad", 3 as "fair", 4 as "delicious" and 5 as "very delicious". A higher tastiness score indicated higher preference towards the corresponding saltiness level of the food. After the tasting session, another research assistant told the participants which ones were the low-sodium options, and that the lowsodium options were good for their health. The participants were then asked to indicate their willingness to consume the low-sodium content foods on a 5-point Likert scale for soup and bread separately, with 1 as "surely not", 2 as "might not", 3 as "not sure", 4 as "might", and 5 as "surely". A higher score indicated greater willingness to consume the low-sodium option after taking into account the taste of the food and the health benefits. The tastiness score and the willingness score were the primary outcome measures.

\section{Measure of Health Literacy on Salt Intake}

The 49-item validated Chinese Health Literacy Scale for Low Salt Consumption - Hong Kong population (CHLSalt-HK) was adopted to measure health literacy on salt intake.[30] The following eight broad areas were covered: functional literacy (term recognition and nutrition label reading), knowledge of the salt content of foods, knowledge of the diseases related to high salt intake, knowledge of international standards, myths about salt intake, attitudes toward salt intake, salty food consumption practices, and nutrition label reading practices. For 5-point Likert scale items, a score of 2 was assigned to the most favourable option, 1 to the next favourable option, and 0 to the remaining options. For 4-option multiple-choice items, a score of 2 was assigned to the correct answer, and 0 to the remaining options. The total score was calculated by summing up the item scores, and the scale had a possible range from 0 to 98 . A higher score indicating higher health literacy related to salt intake.

\section{Procedure}

The study participants were recruited from elderly centres and community centres in Hong Kong from June to August 2014. Trained research assistants explained the study to the potential participants, screened for eligibility, and obtained informed consent. Partly modelled on the study by de Souza et al.,[23] a saltiness preference test was conducted. Both the responsible research assistants and the participants were blinded to the saltiness content of the foods. The participants were interviewed individually so as to minimize peer influence on their food preference. The participants tasted the three options of soups before the three options of bread. The low-sodium, medium-sodium, and high-sodium options were offered in random order. After tasting one of the soups, the participants rated the taste of 
the food according to the 5-point Likert scale described above. Then, the participant rinsed their month with water for at least 30 seconds before tasting the next option. After tasting and rating all the soup options, the participants rinsed their mouth before tasting and rating the three options of bread in the same manner. The participants were allowed to spit the soup or bread after tasting if they did not wish to consume the food due to whatever reasons. The participants who rated the tasted food as "very bad" or "bad" were asked to explain the reason, so as to investigate whether the reasons were related to saltiness or not. The participants also guessed the salt content (low, medium, high) of the tasted foods.

After the tasting session, a different research assistant told the participants which ones were the lowsodium options, and that the low-sodium option was good for their health. Then, the participants rated their willingness to consume the low-sodium option of foods based on the 5-point Likert scale described above. The participants were prompted for the reason if they were not willing to consume the lowsodium option. Finally, the participants responded to the CHLSalt-HK questionnaire and provided demographic information, including age, sex, and presence of chronic illnesses.

\section{Statistical Analysis}

Descriptive statistics were used to summarise the demographic characteristics of the participants. Generalised linear mixed models were used because it could take into account the correlation resulting from the repeated responses from the same individual. Generalised linear mixed models were used to fit linear regressions of tastiness rating for soup and bread separately. To analyse the effect of groups and levels of sodium content on the tastiness rating, tastiness rating was used as the dependent variable, groups of participants and levels of sodium content were used as fixed effects. Random effect was used to capture the correlation between different tastiness ratings from the same respondent. Similarly, generalised linear mixed models were used to fit logistic regressions of probability of the correct identification of sodium content for soup and bread separately. To analyse the effect of groups of participants and levels of sodium content on the correct identification, the binary variable of whether the respondent made a correct identification was used as dependent variable, groups and levels of sodium content were used as fixed effects. Random effect was used to capture the correlation between different responses from the same respondent. To identify factors to the willingness rating to consume the low-sodium option of foods, multiple linear regressions were used with groups of participants, tastiness rating of the low-sodium option, and health literacy about low salt intake as independent variables and the willingness rating as dependent variable. Regression models were fitted for soup and bread separately. SPSS version 20.0 was used. A significance level of $5 \%$ was adopted. The reasons of rating a food option as bad or very bad taste and the reasons of not willing to consume the low-sodium option were also tabulated.

\section{Results}

Sixty hypertensive older people, 49 non-hypertensive older people and 60 non-hypertensive young people participated in the study. Table 1 shows the characteristics of the three groups of participants. The three groups significantly differed $(p<0.001)$ in age, systolic blood pressure, education level, 
occupation, previous diagnosis of hypertension by medical doctor, responsibility of cooking at home, and responsibility of purchasing cooking ingredients. Table 2 shows the mean tastiness ratings of soup and bread with different sodium levels, and Table 3 shows the mean willingness ratings of consuming the low-sodium options of soup and bread. Figure 1 shows the percentage of participants who could correctly identify the sodium content of the food tasted.

Table 4 shows estimated fixed coefficients of generalised linear mixed models of the tastiness ratings of consuming different options of soup and bread. Linear mixed models showed that tastiness rating for high-sodium option of soup was significantly lower than the medium-sodium option $(-1.047,95 \%$ confidence interval $(\mathrm{Cl}):-1.228$ to $-0.867, \mathrm{p}<0.001)$, but there was no significant difference between the low-sodium and the medium-sodium options $(p=0.204)$. For bread, tastiness rating of the low-sodium option and the high-sodium option were significantly lower than the medium-sodium option (-0.379, $95 \% \mathrm{Cl}:-0.546$ to $-0.211, \mathrm{p}<0.001$ for low-sodium option and $-0.302,95 \% \mathrm{Cl}:-0.445$ to $-0.159, \mathrm{p}<0.001$ for high-sodium option). For soup, there was no significant difference in tastiness rating across the groups $(p=0.181$ ). For bread, regardless of the sodium options, the hypertensive older adults and the non-hypertensive older adults gave higher tastiness ratings than non-hypertensive young adults by 0.298 (95\% Cl: 0.065 to $0.531, p=0.012)$ units and 0.325 ( $95 \% \mathrm{Cl}: 0.096$ to $0.553, p=0.006)$ units, respectively. However, there was insignificant difference between the hypertensive older adults and non-hypertensive older adults $(p=0.838)$. Qualitative feedback showed that tastes were the dominant reasons for preference of the food options. Of the 132 reasons for rating the soup as bad or very bad, $90(68 \%)$ complained about the soup being too salty, $12(9 \%)$ about strong taste in general, 10(8\%) about bland taste, $9(7 \%)$ about too sweet, $8(6 \%)$ about poor taste (other than salty or sweet), and the remaining $3(2 \%)$ about poor taste in general. Of the 95 reasons for rating the bread as bad or very bad, $37(39 \%)$ complained the bland taste, 30(32\%) about too salty, $8(8 \%)$ about too sweet, $8(8 \%)$ about their personal dislike of bread, $7(7 \%)$ about poor taste (other than salty or sweet), and the remaining $5(5 \%)$ about texture.

Generalised linear mixed model showed that the participants were more likely to correctly identify highsodium soup than medium-sodium soup (Odds Ratio $(\mathrm{OR})=3.495,95 \% \mathrm{Cl}: 2.115$ to $5.775, \mathrm{p}<0.001$ ), but there was no significant difference between the low-sodium and medium-sodium options $(p=0.347)$. For bread, the participants were more likely to correctly identify low-sodium option than medium-sodium soup (OR=4.823, $95 \% \mathrm{Cl}$ : 2.903 to $8.012, \mathrm{p}<0.001$ ), but they were less likely to correctly identify highsodium option than medium-sodium option ( $\mathrm{OR}=0.514,95 \% \mathrm{Cl}: 0.356$ to $0.741, \mathrm{p}<0.001)$. There was no significant difference among the groups both for soup $(p=0.137)$ and bread $(p=0.509)$.

Table 5 shows the fitted regression models of mean willingness rating for consuming the low-sodium option of soup and bread. Multiple linear regressions showed that higher willingness rating to consume the low-sodium option was significantly $(p<0.001)$ associated with higher tastiness rating of the lowsodium option of soup and bread. The willingness ratings of the low-sodium option of soup and bread increased by 0.526 ( $95 \% \mathrm{Cl}: 0.309$ to 0.742$)$ units and 0.577 (95\% $\mathrm{Cl}: 0.373$ to 0.781$)$ units for one unit increase in the tastiness rating in soup and bread respectively. Higher health literacy of low salt intake was also associated with higher willingness rating for both soup $(p=0.041)$ and bread $(p=0.024)$. The 
willingness ratings of the low-sodium option of soup and bread increased by 0.019 ( $95 \% \mathrm{Cl}: 0.001$ to $0.036)$ units and 0.020 (95\% $\mathrm{Cl}: 0.003$ to 0.038$)$ units for one unit increase in the tastiness rating in soup and bread respectively. Hypertensive older adults tended to be more willing to consume low-sodium option of soup than non-hypertensive older adults by 0.774 ( $95 \% \mathrm{Cl}: 0.278$ to $1.269, \mathrm{p}=0.002)$ units in willingness rating, however there was insignificant difference between non-hypertensive older adults and non-hypertensive young adults $(p=0.156)$. For bread, there was insignificant difference in willingness rating to consume low-sodium option across the three groups of participants $(p=0.375)$. Thirty-three participants indicated not willing to consume the low-sodium option of soup. Nine(27\%) claimed the reason was the poor taste in general, $8(24 \%)$ complained about the taste being too sweet, $5(15 \%)$ claimed that the taste was still too strong, 3(9\%) complained the bland taste, $3(9 \%)$ did not like canned soup, and 5(15\%) had other reasons. One of them even pointed out that taste was more important than healthiness. Thirty-six participants indicated not willing to consume the low-sodium option of bread. Ten(28\%) claimed that the bland taste was the reason, $7(19 \%)$ complained about the poor taste in general, 2(6\%) mentioned the taste was too sweet, $1(3 \%)$ mentioned the taste was too strong, 2(6\%) complained about the texture, $12(33 \%)$ reported that they did not like bread, and the remaining $2(6 \%)$ had other reasons.

\section{Discussions}

This study examined the relationship between the relationship between saltiness preference, willingness to consume low-sodium food among hypertensive older people, non-hypertensive older people and non-hypertensive young people. We found that different saltiness preference was observed for different foods. For soup, participants were indifferent towards low-sodium and medium-sodium option, while they rated significantly lower score for the high-sodium option. For bread, participants rated the medium-sodium option significantly higher, but indifferent between low-sodium and high-sodium options. Meanwhile, there was no evidence showing association between saltiness preference and hypertension status. Moreover, older participants gave higher tastiness ratings to all options of bread than the young participants, but this did not apply to soup. The participants were less likely to correctly identify the high-sodium bread, but more likely to correctly identify high-sodium soup, regardless of the groups of the participants. We also found that the willingness to consume low-sodium foods largely depended on the tastiness, and weakly correlated to the health literacy level of low salt consumption. Hypertensive older adults were more willingness to consume low-sodium foods.

Although soup and bread are not the major component in the daily diet among Hong Kong population, they are popular foods consumed from day to day. According to Hong Kong Population-based Food Consumption Survey $2005-2007$, about $68.3 \%$ and $71.9 \%$ of the respondents consumed bread/rolls/buns and soups respectively.[31] Among the consumers, they had a mean daily consumption of $64.50 \mathrm{~g}$ bread/rolls/buns and $237.61 \mathrm{ml}$ soups. The mean sodium level of plain bun was about $350.0 \mathrm{mg} / 100 \mathrm{~g}$ (range 290.0 to 420.0 ) and that of Chinese soup was about $207.5 \mathrm{mg} / 100 \mathrm{~g}$ (range 190.0 to 240.0).[11] The indifferent tastiness rating of soup between the low-sodium and medium-sodium option implied that there is room for reducing the sodium content of soup to below $120 \mathrm{mg}$ sodium per $100 \mathrm{~g}$ food without significantly affect the tastiness of soup. This could form a public health education message to 
the public to reduce salt added to the soup, no matter during cooking or at table. The tastiness rating scores dropped significantly if the sodium content of bread deviated from the medium level. Reducing the sodium content of bread may face more challenges. Unlike soup, salt in bread not only enhance the favour, but also enhance the texture of bread. Therefore, reduction of sodium content in bread may not be easily achieved by individuals. Professional inputs from the food industry or the chiefs are needed to come up with a receipt which can reduce the sodium content, but can maintain the taste and texture of the bread. Previous studies showed that a reduction up to $50 \%$ of sodium in bread did not significantly affect the consumption of bread.[32-34] One study even pointed out that if flavour was compensated by potassium chloride and yeast extracts, the sodium reduction of bread could be up to 67\% [33]. Therefore, the reduction of sodium in bread should be done progressively, each time by a small amount. While flavour might be compensated, it should be cautious about the increase of other components like potassium, which may not be suitable for certain groups of patients.

The findings that less participants could correctly identify the high-sodium bread required our attention. This implied that the participants might fail to realise that they are consuming high-sodium breads. For the older people, this may be associated with the declining sensitivity in detecting taste.[18] For the young people, it was uncertain if they were used to consume salty food in daily life so that they failed to detect high salt content. It may also reflect participants' lack of knowledge on the definition of "highsodium". While some participants claimed that the food was a bit salty, they still rated the food as delicious and did not rate it as "high sodium". It is important for people to correctly identify high-sodium foods such that they could avoid them. The perception of high-sodium food is food-specific, and perhaps state specific. Soup is a liquid food whereas bread is a solid food. It might be possible that it is more difficult to detect saltiness in solid foods since a medium is needed to reach the taste bud. In our study, participants could identify high-sodium soup easily, but not the high-sodium bread. Hence, we may also alert the public that the high-sodium content in some foods, such as bread, may not be easily recognized.

Our study revealed that the key to willingness to consume low-sodium food was the tastiness of the food. Qualitative feedback from the participants also reassured that taste was sometimes rated more important than health concern. This was consistent with literature that people who claimed salt alternatives as not giving a good taste did not reduce their salt intake despite knowing the health benefits. [25] Therefore, it is important to improve the taste of low-sodium food. Promoting the use of alternatives like sesames to enhance the favour could be encouraged. Compared with the tastiness score, health literacy for low salt consumption may be having a less prominent, yet significant effect. To maximize the consumption of low-sodium food, every possible measure, including the increase of health literacy level through public education, should be involved.

Our study did not show significant difference in the outcome measures among the three different groups of participants, except the hypertensive older participants were more willing to consume the low-sodium soup. This may be because they have higher motivation to change their lifestyle in order to control their hypertension. This is a good sign. However, it should be emphasized that healthy lifestyle should be started before the appearance of diseases. A majority of older people, regardless of their hypertension status, were the main person who cooked for the family and purchased food for the family. Their preference of saltiness would likely affect the whole household instead of merely themselves. 
There was no evidence from our study that the older people were less sensitive to tasting saltiness. This was also a good sign that at least the older people were not preferring saltier foods than the younger people, and hence would be less likely to cook salty food for their families.

There were some limitations of this study. First, this study recruited a convenience sample, which may be different from the general population. In the recruitment process, there were difficulties in recruiting non-hypertensive older people. Some potential participants reported no prior diagnoses of hypertension, yet they had high blood pressure measurements during the screening (after 15-minutes rest before taking measurement). It was uncertain if there are possible "white coat hypertension" or they were undiagnosed hypertension cases. In the study design, while random order of different saltiness level of the foods was adopted, the participants tasted the three options of soups before the three options of bread. There could be potential influence of the difference of taste order between soups and bread.

Some participants reported that they disliked certain types of food (e.g. canned foods or bread), which may be confounder in our study. Hence, a sensitivity analysis was performed by excluding participants who provided reasons unrelated to the salt content in the food. While some participants commented about the sweetness, other tastes or texture of foods, these could be affected by the amount of salt added to the food. Therefore, we excluded 8 participants who mentioned dislike of canned soup or provided other reasons in the analysis of willingness to consume low-sodium soup. Similarly, we excluded 14 participants who mentioned dislike of bread or provided other reasons in the analysis of willingness to consume low-sodium bread. Also, we excluded 4 participants who mentioned dislike of bread ( 8 reasons in total) in any of the three tastings and ran the analysis on tastiness ratings of bread. All these results were consistent with those from the full data.

This study is of practical significance. The Hong Kong population is aging rapidly, with a high prevalence of hypertension, it is expected that the disease burden of cardiovascular disease will be increasing in future. There is an urgent need to promote salt intake reduction. Moreover, interventions have to be tailored for the local settings. The findings helped to inform formulation of the future interventions at the individual level to reduce sodium intake as a means to prevent hypertension. Since people were more concerned about the taste than the health benefits, the focus of future intervention would be demonstration of reducing salt content of food but maintaining the taste. In the long run, we expect that the salt intake of the population will meet the WHO recommendation and thus reducing the prevalence of hypertension and its disease burden.

\section{Acknowledgement:}

The authors wish to thank the colleagues at the elderly centres and the community centres for their assistance during data collection.

\section{Statement of author's contributions to manuscript:}

Chau, Ngai, Leung, Li, Yeung and Tan-Un were responsible for study design, study implementation, writing and final consent of the manuscript. Chau was responsible for statistical analysis. All authors have read and approved the final manuscript. 


\section{Ethical standards:}

Ethics approval had been sought from the Institutional Review Board of the University of Hong Kong/Hospital Authority Hong Kong West Cluster.

Sources of Financial Support:

This study is funded by the Small Project Funding (no. 201309176212) of The University of Hong Kong.

\section{References}

1. Graudal N, Jurgens G, Baslund B, Alderman MH. Compared with usual sodium intake, low- and excessive-sodium diets are associated with increased mortality: a meta-analysis. Am J Hypertens 2014;27(9):1129-37.

2. Brown IJ, Tzoulaki I, Candeias V, et al. Salt intakes around the world: implications for public health. Int J Epidemiol 2009;38(3):791-813.

3. World Health Organization. Prevention of Cardiovascular Disease: Guidelines for Assessment and Management of Total Cardiovascular Risk. Geneva: World Health Organization, 2007.

4. He FJ, MacGregor GA. Reducing population salt intake worldwide: from evidence to implementation. Prog Cardiovasc Dis 2010;52(5):363-82.

5. Mozaffarian D, Benjamin EJ, Go AS, et al. Heart disease and stroke statistics--2015 update: a report from the American Heart Association. Circulation 2015;131(4):e29-322.

6. He FJ, Li J, Macgregor GA. Effect of longer-term modest salt reduction on blood pressure. Cochrane Database Syst Rev 2013;4:Cd004937.

7. Mohan S, Campbell NR. Salt and high blood pressure. Clin Sci (Lond) 2009;117(1):1-11.

8. Chau PH, Woo J, Goggins WB, Tse YK, Chan KC, Lo SV, Ho SC. Trends in stroke incidence in Hong Kong differ by stroke subtype. Cerebrovasc Dis 2011;31(2):138-46.

9. Census and Statistics Department, The Government of the Hong Kong Special Administrative Region. 2011 Population Census. Main Report: Volume I. Hong Kong, 2012.

10. The Chinese University of Hong Kong. Increasing Salt Intake Predisposes Hong Kong People to Hypertension and Its Complications 2005. http://www.cuhk.edu.hk/ipro/pressrelease/050511e.htm.

11. Centre for Food Safety, Food and Environmental Hygiene Department, The Government of the Hong Kong Special Administrative Region. The First Hong Kong Total Diet Study: Minerals. The First Hong Kong Total Diet Study Report 2014;9:1-131.

12. Liu ZM, Ho SC, Tang N, Chan R, Chen YM, Woo J. Urinary sodium excretion and dietary sources of sodium intake in chinese postmenopausal women with prehypertension. PLoS One 2014;9(8):e104018.

13. Task Force on Conceptual Model and Preventive Protocols, Woring Group on Primary Care, Food and Health Bureau. Hong Kong Reference Framework for Hypertension Care for Adults in Primary Care Settings. 2010. http://www.pco.gov.hk/english/resource/files/RF_HT_full.pdf.

14. Dunford EK, Eyles H, Mhurchu CN, Webster JL, Neal BC. Changes in the sodium content of bread in Australia and New Zealand between 2007 and 2010: implications for policy. Med J Aust 2011;195(6):346-9.

15. Ferrante D, Apro N, Ferreira V, et al. Feasibility of salt reduction in processed foods in Argentina. Rev Panam Salud Publica 2011;29(2):69-75.

16. Newson RS, Elmadfa I, Biro G, et al. Barriers for progress in salt reduction in the general population. An international study. Appetite 2013;71:22-31. 
17. Stevens JC, Cain WS. Changes in taste and flavor in aging. Crit Rev Food Sci Nutr 1993;33(1):27-37.

18. Nordin S, Razani LJ, Markison S, Murphy C. Age-associated increases in intensity discrimination for taste. Exp Aging Res 2003;29(3):371-81.

19. Hayes JE, Sullivan BS, Duffy VB. Explaining variability in sodium intake through oral sensory phenotype, salt sensation and liking. Physiol Behav 2010;100(4):369-80.

20. Shepherd R, Farleigh CA, Wharf SG. Limited compensation by table salt for reduced salt within a meal. Appetite 1989;13(3):193-200.

21. Piovesana PdM, Sampaio KdL, Gallani MCBJG. Association between taste sensitivity and selfreported and objective measures of salt intake among hypertensive and normotensive individuals. ISRN Nutr 2013;2013:301213.

22. Olayemi SO, Mabadeje AF. Comparative study of salt taste threshold of hypertensives, their normotensive relatives and non-relatives. Niger Postgrad Med J 2003;10(2):96-8.

23. de Souza JT, Matsubara LS, Menani JV, Matsubara BB, Johnson AK, De Gobbi JI. Higher salt preference in heart failure patients. Appetite 2012;58(1):418-23.

24. Van der Veen JE, De Graaf C, Van Dis SJ, Van Staveren WA. Determinants of salt use in cooked meals in The Netherlands: attitudes and practices of food preparers. Eur J Clin Nutr 1999;53(5):388-94.

25. Smith SL, Quandt SA, Arcury TA, Wetmore LK, Bell RA, Vitolins MZ. Aging and eating in the rural, southern United States: beliefs about salt and its effect on health. Soc Sci Med 2006;62(1):18998.

26. Nagata C, Sugiyama C, Shimizu H. Nutrient intakes in relation to style of breakfast and taste preferences. J Epidemiol 1999;9(2):91-8.

27. Zallen EM, Hooks LB, O'Brien K. Salt taste preferences and perceptions of elderly and young adults. J Am Diet Assoc 1990;90(7):947-50.

28. Mattes RD, Kumanyika SK, Halpern BP. Salt taste responsiveness and preference among normotensive, prehypertensive and hypertensive adults. Chem Senses 1983;8(1):27-40.

29. Pangborn RM, Pecore SD. Taste perception of sodium chloride in relation to dietary intake of salt. Am J Clin Nutr 1982;35(3):510-20.

30. Chau PH, Leung AY, Li HL, Sea M, Chan R, Woo J. Development and Validation of Chinese Health Literacy Scale for Low Salt Consumption - Hong Kong Population (CHLSalt-HK). PLoS One 2015;10(7):e0132303.

31. Food and Environmental Hygiene Department, The Government of the Hong Kong Special Administrative Region. Hong Kong Population-based Food Consumption Survey 2005-2007. Hong Kong, 2010.

32. La Croix KW, Fiala SC, Colonna AE, et al. Consumer detection and acceptability of reduced-sodium bread. Public Health Nutr 2015;18(8):1412-8.

33. Bolhuis DP, Temme EH, Koeman FT, et al. A salt reduction of $50 \%$ in bread does not decrease bread consumption or increase sodium intake by the choice of sandwich fillings. J Nutrition 2011;141(12):2249-55.

34. Girgis S, Neal B, Prescott J, et al. A one-quarter reduction in the salt content of bread can be made without detection. Eur J Clin Nutr 2003;57(4):616-20. 
Table 1 Characteristics of participants

\begin{tabular}{|c|c|c|c|}
\hline Characteristics & $\begin{array}{c}\text { Hypertensive } \\
\text { older adults } \\
(n=60)\end{array}$ & $\begin{array}{c}\text { Non-hypertensive } \\
\text { older adults } \\
(n=49)\end{array}$ & $\begin{array}{c}\text { Non-hypertensive } \\
\text { young adults } \\
(n=60)\end{array}$ \\
\hline Age (years), mean $\pm S D^{*}$ & $76.3 \pm 6.5$ & $72.0 \pm 8.8$ & $29.4 \pm 8.1$ \\
\hline $\begin{array}{l}\text { Systolic blood pressure }{ }^{\circledR} \text {, mean } \pm S D^{*} \\
\text { Diastolic blood pressure }{ }^{\circledR} \text {, mean } \pm S D \\
\text { CHLSalt-HK score, mean } \pm S D\end{array}$ & $\begin{array}{l}141.8 \pm 18.7 \\
76.4 \pm 12.0 \\
62.2 \pm 11.9\end{array}$ & $\begin{array}{c}125.6 \pm 12.5 \\
73.4 \pm 8.4 \\
65.8 \pm 11.5\end{array}$ & $\begin{array}{c}110.5 \pm 12.5 \\
71.8 \pm 9.4 \\
60.8 \pm 10.1\end{array}$ \\
\hline CHLSalt-HK score, mean \pm SD & Count (\%) & Count (\%) & Count (\%) \\
\hline \multicolumn{4}{|l|}{ Sex } \\
\hline Male & $11(18.3)$ & $9(18.4)$ & $15(25.0)$ \\
\hline Female & $49(81.7)$ & $40(81.6)$ & $45(75.0)$ \\
\hline \multicolumn{4}{|l|}{ Educational Level* } \\
\hline Primary or below & $23(39.0)$ & $15(33.3)$ & $0(0.0)$ \\
\hline Secondary & $22(37.3)$ & $20(44.4)$ & $2(10.0)$ \\
\hline Tertiary or above & $14(23.7)$ & $10(22.2)$ & $18(90.0)$ \\
\hline \multicolumn{4}{|l|}{ Occupation* } \\
\hline Retired & $51(85.0)$ & $37(75.5)$ & $0(0.0)$ \\
\hline Home-maker & $8(13.3)$ & $9(18.4)$ & $1(1.7)$ \\
\hline Students & $0(0.0)$ & $0(0.0)$ & $13(21.7)$ \\
\hline Economically Active & $1(1.7)$ & $3(6.1)$ & $46(76.7)$ \\
\hline \multicolumn{4}{|l|}{ Vegetarian } \\
\hline Yes (Daily or regularly) & $9(15.0)$ & $6(12.2)$ & $5(8.3)$ \\
\hline No & $51(85.0)$ & $43(87.8)$ & 55 (91.7) \\
\hline \multicolumn{4}{|l|}{$\begin{array}{l}\text { Previous diagnosis of hypertension by } \\
\text { medical doctor* }\end{array}$} \\
\hline Yes & 46 (76.7) & $0(0.0)$ & $0(0.0)$ \\
\hline No & $14(23.3)$ & $49(100.0)$ & $60(100.0)$ \\
\hline \multicolumn{4}{|l|}{ Main person who cook for the family* } \\
\hline Yes & $40(66.7)$ & $33(67.3)$ & $15(25.0)$ \\
\hline No & $20(33.3)$ & $16(32.7)$ & $45(75.0)$ \\
\hline \multicolumn{4}{|l|}{$\begin{array}{l}\text { Main person who purchase cooking } \\
\text { ingredients for the family* }\end{array}$} \\
\hline Yes & $43(71.7)$ & $37(75.5)$ & $15(25.0)$ \\
\hline No & $17(28.3)$ & $12(24.5)$ & $45(75.0)$ \\
\hline
\end{tabular}

Note: * significant difference among the groups at $5 \%$ level of significance; @ based on the average value from two measurements. 
Table 2 Mean (Standard Deviation) of the tastiness ratings of soup and bread with different sodium levels

\begin{tabular}{|l|c|c|c|c|c|c|}
\hline Groups & \multicolumn{3}{|c|}{ Soup } & \multicolumn{3}{c|}{ Bread } \\
& Low & Medium & High & Low & Medium & High \\
\hline \multirow{2}{*}{ Hypertensive older adults $(\mathrm{n}=60)$} & 3.483 & 3.600 & 2.633 & 3.317 & 3.550 & 3.200 \\
& $(1.000)$ & $(0.848)$ & $(1.178)$ & $(0.948)$ & $(0.964)$ & $(1.022)$ \\
\hline \multirow{2}{*}{ Non-hypertensive older adults $(\mathrm{n}=49)$} & 3.408 & 3.531 & 2.510 & 3.122 & 3.673 & 3.388 \\
& $(0.864)$ & $(1.120)$ & $(1.102)$ & $(0.971)$ & $(0.826)$ & $(1.077)$ \\
\hline \multirow{2}{*}{ Non-hypertensive young adults $(\mathrm{n}=60)$} & 3.333 & 3.433 & 2.283 & 2.900 & 3.283 & 3.017 \\
& $(0.877)$ & $(0.722)$ & $(0.993)$ & $(0.951)$ & $(0.691)$ & $(0.813)$ \\
\hline
\end{tabular}

Table 3 Mean (Standard Deviation) of the willingness ratings of consuming low-sodium option of soup and bread

\begin{tabular}{|l|c|c|}
\hline Groups & Soup & Bread \\
\hline Hypertensive older adults $(n=60)$ & $4.400(1.291)$ & $4.267(1.247)$ \\
\hline Non-hypertensive older adults $(n=49)$ & $3.755(1.640)$ & $3.898(1.475)$ \\
\hline Non-hypertensive young adults $(n=60)$ & $3.950(1.254)$ & $3.817(1.467)$ \\
\hline
\end{tabular}

Table 4 Estimated fixed coefficients of generalised linear mixed models of the tastiness ratings of consuming different options of soup and bread

\begin{tabular}{|c|c|c|c|}
\hline \multirow{2}{*}{\begin{tabular}{|l|} 
Fixed factors \\
Intercept \\
\end{tabular}} & $\begin{array}{c}\text { Soup } \\
\text { Coefficient }(95 \% \mathrm{Cl})\end{array}$ & \multicolumn{2}{|c|}{$\begin{array}{c}\text { Bread } \\
\text { Coefficient }(95 \% \mathrm{Cl}) \\
\end{array}$} \\
\hline & $3.417^{*}(3.258,3.575)$ & $3.291^{*}$ & $(3.132,3.451)$ \\
\hline \multicolumn{4}{|l|}{ Group } \\
\hline Hypertensive older adults & $0.199(-0.015,0.412)$ & $0.298 *$ & $(0.065,0.531)$ \\
\hline Non-hypertensive older adults & $0.116(-0.142,0.373)$ & $0.325 *$ & $(0.096,0.553)$ \\
\hline Non-hypertensive young adults & 0 & 0 & \\
\hline \multicolumn{4}{|l|}{ Sodium level } \\
\hline Low & $-0.112(-0.286,0.061)$ & $-0.379 *$ & $(-0.546,-0.211)$ \\
\hline High & $-1.047^{*}(-1.228,-0.867)$ & $-0.302^{*}$ & $(-0.445,-0.159)$ \\
\hline Medium & 0 & 0 & \\
\hline
\end{tabular}

Note: $\mathrm{Cl}=$ Confidence Interval; ${ }^{*}=$ significantly different from the reference group $(p<0.05)$ 
Table 5 Estimated coefficients of multiple linear regressions of the willingness ratings of consuming the low-sodium option of soup and bread

\begin{tabular}{|c|c|c|}
\hline Factors & $\begin{array}{c}\text { Soup } \\
\text { Coefficient }(95 \% \mathrm{Cl})\end{array}$ & $\begin{array}{c}\text { Bread } \\
\text { Coefficient }(95 \% \mathrm{Cl})\end{array}$ \\
\hline Intercept & $1.071(-0.329,2.471)$ & $0.912 \quad(-0.293,2.118)$ \\
\hline \multicolumn{3}{|l|}{ Group } \\
\hline Hypertensive older adults & $0.414(-0.051,0.879)$ & $0.173 \quad(-0.292,0.638)$ \\
\hline Non-hypertensive older adults & $-0.359(-0.858,0.139)$ & $-0.175 \quad(-0.667,0.317)$ \\
\hline Non-hypertensive young adults & 0 & 0 \\
\hline Tastiness rating of low-sodium option & $0.526 * \quad(0.309,0.742)$ & $(0.373,0.781)$ \\
\hline CHLSalt-HK Score & $0.019 * \quad(0.001,0.036)$ & $(0.003,0.038)$ \\
\hline
\end{tabular}

Note: $\mathrm{Cl}=$ Confidence Interval; * = significantly different from the reference group $(p<0.05)$;

CHLSalt-HK = Chinese Health Literacy Scale for Low Salt Consumption-Hong Kong Population

Figure 1 Percentage of participants who could correctly identified the sodium content of the food tasted

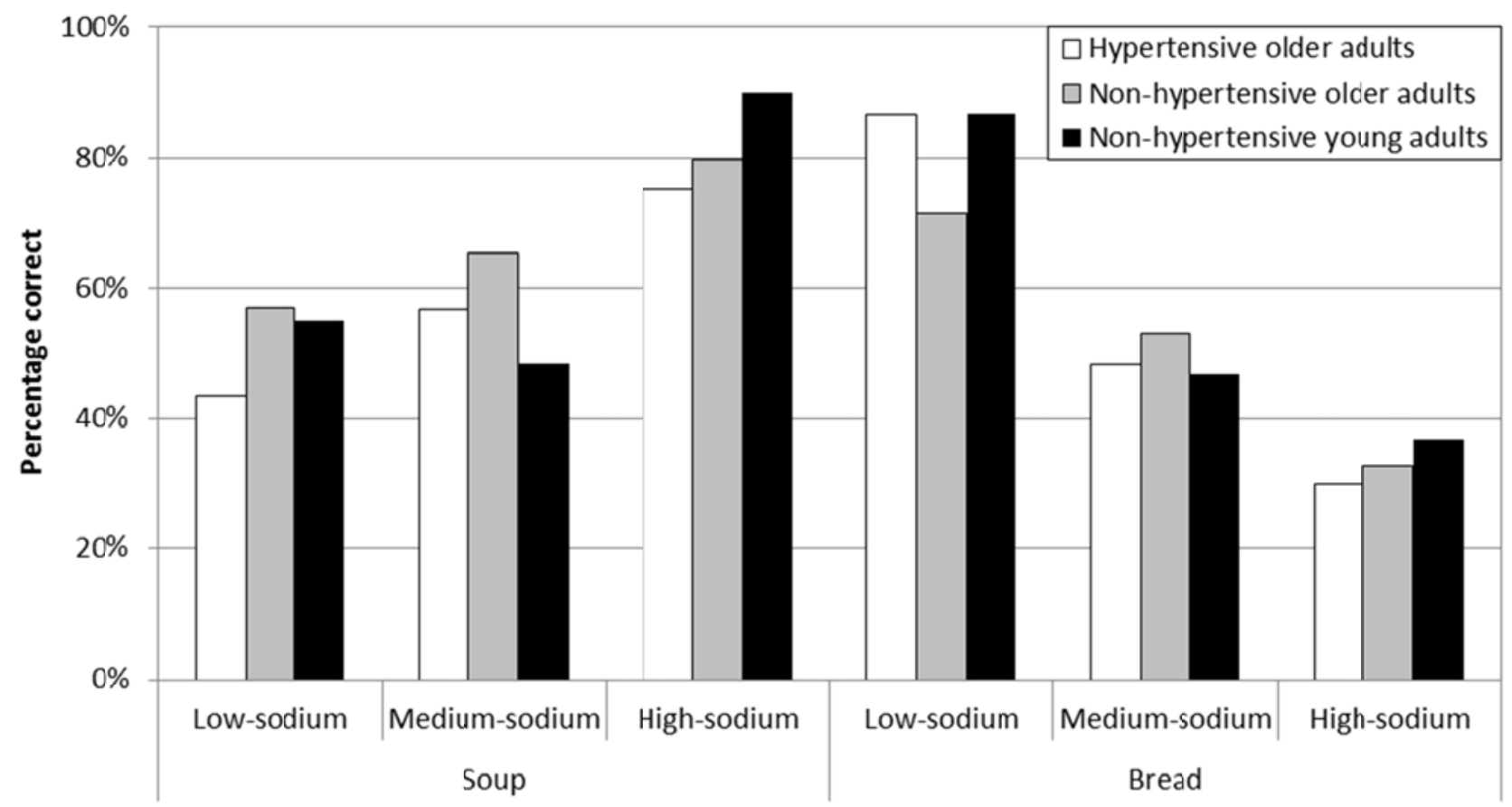

